\title{
A multicolour view of the nuclei of dEs
}

\section{Thorsten Lisker, Eva K. Grebel and Bruno Binggeli}

Astronomical Institute, University of Basel, Switzerland; email: tlisker@astro.unibas.ch

\begin{abstract}
Using a sample of over 100 nucleated Virgo cluster dEs from the SDSS we show that interchanging nucleus and galaxy colours does not alter the colour-magnitude relation. No significant difference is found between nuclei and their parent galaxies in a multicolour comparison except for a weak tendency that some nuclei are slightly redder than their hosts.
\end{abstract}

Keywords. galaxies: nuclei, galaxies: dwarf, galaxies: clusters: general

Nuclei of dwarf ellipticals presumably are compact central star clusters that form a bright extension of globular clusters (GCs) in their sizes and luminosities (Lotz et al. 2004). Their formation process is poorly understood - possible scenarios include early in-situ formation, late star formation out of central gas, or infall and merging of massive GCs via dynamical friction (Oh \& Lin 2000). Photometric studies have been contradictory so far: while Lotz et al. (2004) find from HST/WFPC2 data the nuclei to be slightly bluer than their host galaxies and to be consistent with GC colours, no colour difference was seen in previous ground-based studies.

For 187 Virgo dENs from the SDSS Data Release 3 we performed aperture photometry for a central $\left(r<1.2^{\prime \prime}\right)$ as well as a half-light radius aperture, excluding the inner $r<2^{\prime \prime}$ for the latter. Although the central aperture is intended to represent the nucleus, in most cases the underlying galaxy still dominates the flux as illustrated in the left panel of Fig. 1. We would therefore not be able to see very small differences in nucleus and galaxy colour, but the large sample size and wide colour basis compensate for this to some extent and should unveil any significant systematic differences. Figure 1 (right panel) shows that nucleus and galaxy $\mathrm{u}-\mathrm{z}$ colours agree within the errors - still, a tiny offset towards redder nuclei seems to be present. This offset does not become stronger for larger nucleus-togalaxy ratios (larger symbols), indicating that it might at best apply to a fraction of objects rather than to the full sample. In the colour-magnitude relation (Fig. 2, left panel, using $113 \mathrm{dENs}$ ) nucleus and galaxy colours can be interchanged without altering its shape; only at the very faint end where measurement errors are large, there seems to be a number of slightly redder nuclei. Moreover, several nuclei show redder u-g colours in the colour-colour diagram (Fig. 2, right panel). However, the measurement errors are large compared to the relatively small range of colours and thus prevent any definite statement on the number of these nuclei or on a possible systematic effect applying to all objects. Clearly, high-quality spectroscopy would be the proper way to decide whether or not nuclei and their host galaxies do consist of the same stellar population.

\section{Acknowledgements}

We thank the SDSS collaboration (http://www.sdss.org/) for the wealth of DR3 data.

\section{References}

Lotz, J.M., Miller, B.W. \& Ferguson, H.C. 2004, ApJ 613, 262

Oh, K.S. \& Lin, D.N.C. 2000, ApJ 543, 620 

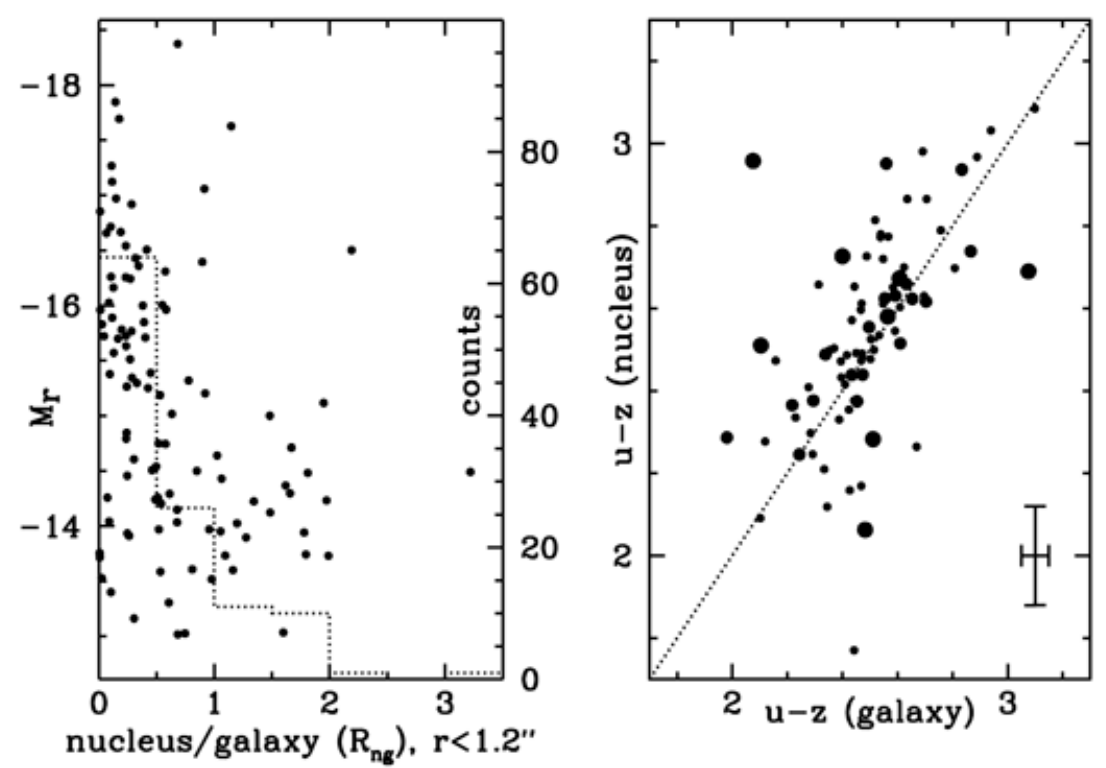

Figure 1. Left: Absolute galaxy magnitude in the $\mathrm{r}$ band (adopting $m-M=31$ ) versus the flux ratio of nucleus to galaxy within $r<1.2^{\prime \prime}$. The dotted line shows a histogram with number counts given on the right y-axis. Right: Comparison of nucleus and galaxy u-z colours. Large symbols represent objects with a nucleus-to-galaxy ratio $R_{n g} \geqslant 1$, intermediate symbols stand for $0.5 \leqslant R_{n g}<1$, and small symbols mean $R_{n g}<0.5$. Only objects brighter than $m_{r} \leqslant 16$ were selected to minimize measurement uncertainties. The dotted line stands for equality.

Nucleus (•) vs. host galaxy (•) colours
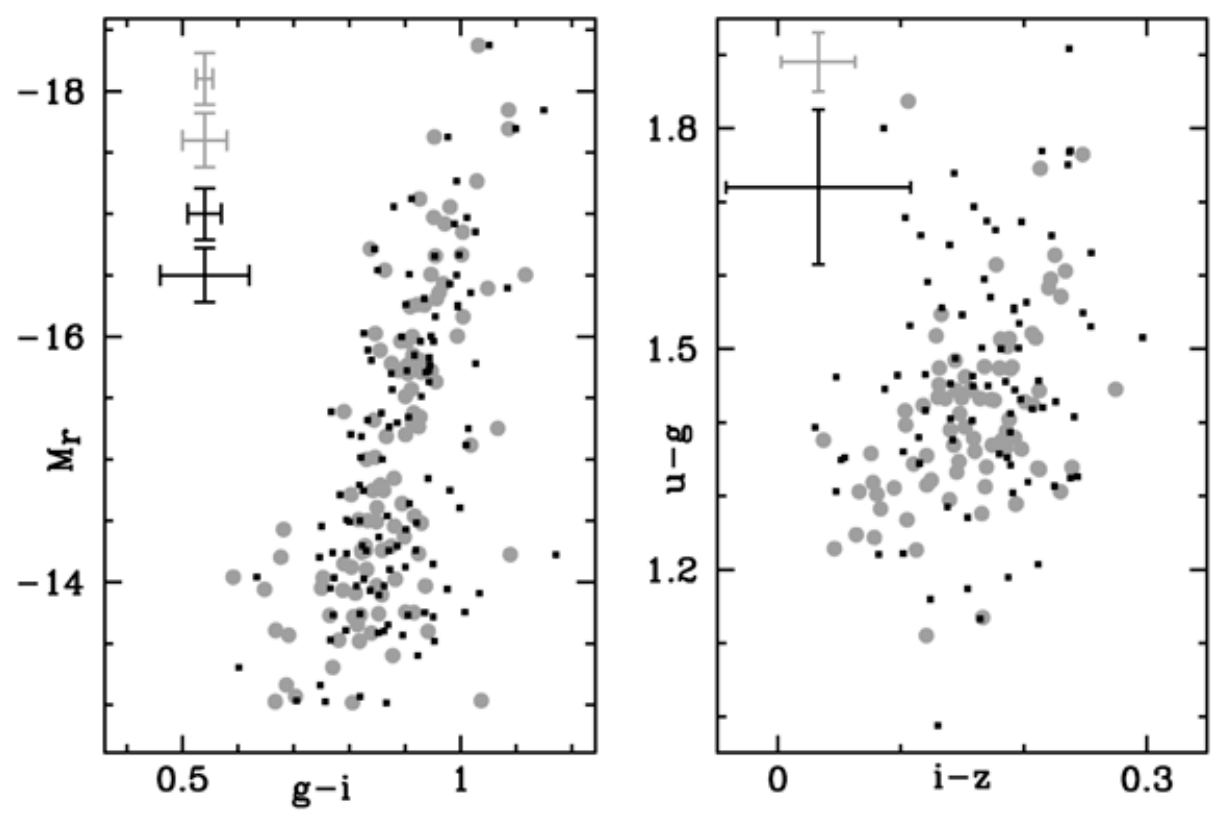

Figure 2. Left: Absolute galaxy magnitude in the $\mathrm{r}$ band $(m-M=31)$ versus galaxy colours (circles) and nucleus colours (squares). Colours were measured from half-light radius and central $\left(r<1.2^{\prime \prime}\right)$ apertures, respectively. Upper error bars are for the brighter part of the sample, lower error bars for the fainter part. Right: Colour-colour plot; symbols as in the left panel. Only objects brighter than $m_{r} \leqslant 16$ were selected to minimize measurement uncertainties. 